\title{
Effect of some safety compounds and application method on growth and productivity of globe artichoke (Cynara scolymus, l.)
}

\author{
Abd El Halem .M. K. ${ }^{1}$; Nadia S. Shafshak ${ }^{2}$ F. A.Abo-Sedera ${ }^{2}$; Afaf T. Kasim ${ }^{1}$ and A. S. Shams ${ }^{2}$. \\ 1- Potato and Vegetatively Propagated Vegetables Department, Hort. Res., Institute, Egypt. \\ 2- Hort. Dept., Fac. of Agric., Benha Univ., Egypt. \\ Corresponding author: abdelhakeem.shams@fagr.bu.edu.eg
}

\begin{abstract}
Two field experiments were conducted during two successive seasons of 2014/2015 and 2015/2016 at Vegetable Experimental Research Farm in Kaha, Kaliobia Governorate to study the effect of application method of safety compounds on growth and productivity of globe artichoke cv. "Concerto" used treatments included three different application method; i.e., soaking 20 min., spraying 6 times started after 70 days from planting date and repeated every 15 days through the growth season and soaking plus spraying in seven different safety compounds; i.e., Amino power (source of free amino acids) $0.5 \mathrm{~cm}^{3} / 1$, Fruiting (source of gibberellins) $0.5 \mathrm{~cm}^{3} / 1$, Mega humic acid (source of humic acid) $0.25 \mathrm{~g} / 1$, Cytojeep (source of gibberellins plus free amino acids) $0.5 \mathrm{~cm}^{3} / 1$, Chitosan $1 \%$ and Garlic extract $50 \mathrm{~cm}^{3} / 1$ as well as tap water (control). A split plot design with four replicates was used in this experiment. Main plots were application method and sub-plots were safety compounds.

The obtained results revealed that spraying Mega humic acid or soaking plus spraying significantly produced the highest leaves number/ plant, plant height, produced yield (early, late and total) expressed as heads weight / plant, head and edible part weight (early and late yield), dry matter percentage in late yield as well as the lowest total phenols in early yield comparing with the control. On the other hand, using Cytojeep significantly produced the highest offshoots number / plant and the least number of days to the anthesis. Also, spraying Fruiting significantly increased inuline percentage in early and late yield and decreased the fibers contents in late yield.
\end{abstract}

Key words: Cynara scolymus L, Humic acid, Garlic extract, Chitosan, gibberellins, Soaking, Spraying

\section{Introduction}

Globe artichoke (Cynara scolymus L.) belongs to composite family. It is one of the most important vegetable crops grown in Egypt for local markets and exportation. The period of artichoke exporting has been extended throughout the months of the year, and this calls for the use of new varieties whose production extends longer than those of local varieties. The following factors amino acids, humic acid, garlic extracts and chitosan as well as gibberellins have received the most attention to stimulate and enhance growth and productivity of many vegetable crops and its contents of high levels of organic matter, essential macro and micro nutrients and vitamins.

Additionally, the role of amino acids in broccoli plants is the activation of photosynthesis and activates the formation of chlorophyll as well as increase the vegetative growth and early yield (Shekari and Javan Mardi 2017). While, gibberellins are plant hormones that regulate growth and influence various developmental processes, including stem elongation, germination, dormancy, flowering, sex expression, enzyme induction, and leaf and fruit senescence (Scott, 2008). Moreover, The main role of garlic extract in its effect on plant diseases with increases a vital resistance of potato plants and improve plant healthy (Al Mayahi and Fayadh 2016). The direct effects of humic acid compounds may have various biochemical effects either at cell wall, membrane level or in the cytoplasm, including increasing photosynthesis and respiration rates in plants, enhancing protein synthesis and plant hormone like activity (Akinremi et al. 2000). Meanwhile, many investigators reported that using chitosan as foliar spray increasing vegetative growth, yield and quality of some vegetable crops. (Shams et al. 2014; El-Sagan and El-Dsouky 2015; and El-Adawy 2017). Therefore, the present study aimed to investigate the effect of application method with amino acids, gibberellins, humic acid, garlic extract and chitosan levels on vegetative growth, earliness, yield and quality of globe artichoke heads.

\section{Materials and Methods}

Two field experiments were conducted at Vegetable Experimental Research Farm in Kaha, Kaliobia Governorate, during of 2014/2015 and $2015 / 2016$ seasons to study the effect of some safety compounds i.e, Cytojeep, Amino Power, Fruiting, Mega Humic Acid, Garlic Extract and Chitosan added as foliar spraying or soaking as well as their combination on earliness and productivity of Globe artichoke cv. Concerto (Cynara scolymus L). The plant materials of the used cultivar were obtained from Horticulture Research Institute, Agriculture Research Center, Ministry of Agriculture.

The soil type of the experimental site was clay with $\mathrm{pH}$ of 8.1 and Ec.1.93 dSm as average of both seasons.

The experimental design was a split plot design with four replicates. The experiment included the following treatments as: 
Main plots were for application methods (3 treatments) as foliar spraying six times started after 70 days from planting date and repeated every 15 days through the growth season and soaking for $20 \mathrm{~min}$. as well as interaction between them.

The sub-plots were assigned to: control (tap water), Amino power $0.5 \mathrm{~cm}^{3} / 1$, Cytojeep $0.5 \mathrm{~cm}^{3} / 1$, Mega humic acid $0.25 \mathrm{~g} / \mathrm{l}$, Fruiting $0.5 \mathrm{~cm}^{3} / 1$, Chitosan $1 \%$ and Garlic extract $50 \mathrm{~cm}^{3} / 1$.

The planting dates were end of August and first of September in the $1^{\text {st }}$ and $2^{\frac{\text { nd }}{}}$ seasons, respectively. The propagation materials were treated pre-planting with fungicides for 30 minutes and hand planted at $1 \mathrm{~m}$ apart between each two plants on the ride and $1 \mathrm{~m}$ between the ridges, plot area was $20 \mathrm{~m}^{2}$ (4 lines $\times 5 \mathrm{~m}$ long $\times 1 \mathrm{~m}$ width). Pest control and other agriculture practices were applied as commonly recommended for commercial globe artichoke production by Ministry of Agriculture.
A commercial Amino power consists of (free amino acids $19 \%$, micro elements $1500 \mathrm{ppm}$ and potassium citrate $3.5 \%$ ). Cytojeep (free amino acids, $\mathrm{GA}_{3} 5 \%$, nitrogen $4 \%$, cytokinin $1.5 \%$ and potassium oxide $3 \%$ ). Mega humic acid (humic acid $92 \%$ and potassium humat $8 \%$ ). Fruiting (free amino acids, boron, $\mathrm{GA}_{3}$, citric acid $1 \%$, phosphorus $5 \%$ and potassium oxide $25 \%$ ). Chitosan (2-Amino-2deoxy-beta-D-glucosasmine) solution was prepared by dissolving a proper amount of Chito-Care ${ }^{\circ}$, (an Egyptian commercial product of chitosan) in $1 \%$ acetic acid solution.

The usual cultural procedures of globe artichoke growing including fertilization which was done using $350 \mathrm{~kg}$ ammonium sulphate, $200 \mathrm{~kg}$ calcium super phosphate and $200 \mathrm{~kg}$ potassium sulphate /fed. were added in three equal doses after 2,3 and 4 months from planting.

Table A. Some chemical constituents of garlic cloves analyzed in Arid Land Agricultural Research unit.

\begin{tabular}{cc}
\hline Components & Concentration \\
\hline GA3 & $1.633 \mathrm{mg} / 100 \mathrm{~g} \mathrm{~F} . \mathrm{W}$. \\
\hline $\mathrm{IAA}$ & Trace amount \\
\hline $\mathrm{ABA}$ & Trace amount \\
\hline $\mathrm{Ca}$ & $1.363 \%$ \\
\hline $\mathrm{Mg}$ & $1.230 \%$ \\
\hline $\mathrm{SO} 4$ & $0.181 \%$ \\
\hline $\mathrm{Zn}$ & $66.5 \mathrm{ppm}$ \\
\hline $\mathrm{Mn}$ & $94.4 \mathrm{ppm}$ \\
\hline
\end{tabular}

\section{Data recorded:}

\section{1 - Plant survival percentage and germination speed}

a. Plant survival (\%): The survival percentage was counted after 45 days from planting in the permanent field and the survival percentage was estimated according to the following formula:

Survival percentage $=$ Number of survived plants / Number of planted offshoots x 100 .

b. Germination speed $=\{$ ( No. of plants in the first day $\times 1)+($ No. of plants in the second day $\times 2)$ $+\ldots \ldots$ at growth end $\} /$ Total No. of plants

\section{2-Vegetative growth characteristics:}

Five plants were randomly chosen from each subplot and marked to evaluate plant height, leaves number per plant and the offshoots number per plant in the beginning of the anthesis during the two seasons.

3 - Flowering behavior: number of days elapsed from planting to start blooming stage

4 -Flower head yield and its components: early, late and total flower head yield based on weight of heads per plant were recorded as follows a. The early yield: it was expressed as weight of heads produced from the first of March till the middle of April (45 days).

b. The late yield: expressed as weight of heads produced from the middle of April till the end of June end of the production season of globe artichoke (75 days) $\}$.

c. Total yield: expressed as weight of all heads produced throughout the entire harvesting season (120 days).

\section{5- Head characteristics:}

Average head weight and the edible part weight were recorded in monthly yield throughout the harvesting season in a representative sample of 20 flower heads.

\section{6- Head chemical composition:}

For chemical determination, a representative sample of $100 \mathrm{~g}$. from the edible part for each experimental treatment was taken, oven dried at $70{ }^{\circ} \mathrm{C}$ till constant weight then dry matter percentage was recorded, Inulin percentage and total phenolic contents as well as crud fibers were determined. Inulin was assayed according to the method of Winton and Winton (1958). Also, crude fibers content of the edible part was estimated according to the method of A.O.A.C. (2000). Total phenolic contents were 
determined according to the Folin-Ciocalteu method as described by Zheng and Wang (2001).

\section{7- Statistical analysis:}

All obtained data were subjected to the proper analysis according to SAS (1996) and the means were compared using the least significant differences test (L.S.D.) at 5\% (Snedecor and Cochran, 1991).

\section{Results and Discussion}

\section{Plant survival percentage and germination} speed :

Table 1. Effect of pre plant soaking of propagation materials in some plant stimulants on survival plant percentage and germination speed of globe artichoke during 2014/2015 and 2015/2016 seasons

\begin{tabular}{lcccc}
\hline \multirow{2}{*}{ Treatment } & \multicolumn{2}{c}{ Survival percentage } & \multicolumn{2}{c}{ Germination speed (days) } \\
\cline { 2 - 5 } & $\mathbf{2 0 1 4 / 1 5}$ & $\mathbf{2 0 1 5 / 1 6}$ & $\mathbf{2 0 1 4 / 1 5}$ & $\mathbf{2 0 1 5 / 1 6}$ \\
\hline Amino power & 66.25 & 66.67 & 13.78 & 13.94 \\
\hline Fruiting & 55.42 & 69.17 & 15.82 & 15.75 \\
\hline Mega humic acid & 72.92 & 78.33 & 11.96 & 11.81 \\
\hline Chitosan & 41.25 & 41.67 & 17.69 & 18.52 \\
\hline Garlic extract & 68.33 & 72.92 & 12.81 & 13.43 \\
\hline Cytojeep & 57.92 & 60.83 & 15.15 & 15.31 \\
\hline Control & 46.66 & 55.83 & 16.31 & 16.35 \\
\hline L.S.D. at $\mathbf{0 . 0 5}$ & $\mathbf{3 . 2 5}$ & $\mathbf{4 . 2 0}$ & $\mathbf{0 . 6 8}$ & $\mathbf{0 . 8 5}$ \\
\hline
\end{tabular}

L.S.D. = Low significant different.

\section{2- Vegetative growth characteristics:}

Data presented in Table (2) illustrate that soaking plant materials and spraying plants as application method increased significantly plant height in both seasons (2014/2015 and 2015/2016) and offshoots number per plant in the first season only, while there were no significant differences among factors of this study in leaves number per plant during both seasons. Also, it was noticed that using Mega humic acid or Cytojeep increased significantly leaves number per plant and plant height in two seasons as well as offshoots number per plant in first seasons only, meanwhile, in the second season the highest values were due to using Mega humic acid or Garlic extract. These findings agree with those obtained by Shehata et al. (2016) and Mohsen et al. (2017) who mentioned that treating plants with humic acid increased leaves number and plant height in cucumber and garlic plants respectively.

Concerning the interaction effect, data in the same Table indicate that soaking Mega humic acid or plus spraying exhibited the highest values of

leaves number per plant irrespective of significant differences. Also, treated plants with Mega humic acid as a soaking plus spraying led to significant increase in plant height but there were no significant differences among Mega humic acid, Cytojeep and Garlic extract as soaking plus spraying during the second season.

\section{3 -Flowering behavior}

Data in Figures (1) and (2) Show that there was no significant effect due to using either spraying or
Data presented in Table (1) show the effect of pre planting (soaking propagation materials) on plant stimulants solutions on survival percentage and germination speed. Data indicate that soaking propagation materials in Mega humic acid increased significantly survival plant percentage and germination speed compared with other used treatments during the two seasons. These findings agree with those obtained by Sajid $\boldsymbol{e t}$ al. (2012) who found that survival percentage of onion plants increased with high level of humic acid application (3 $\left.\mathrm{kg} \mathrm{ha}^{-1}\right)$. soaking plus spraying. Meanwhile, the lowest number of days elapsed from planting until starting flowering was recorded in case of using soaking plus spraying with significant differences as compared with soaking only. Data in the same Figures show clearly that using Mega humic acid and Cytojeep exhibited significant effect compared with the other treatments. In this regard the most effective treatment which reflected the lowest number of days to the anthesis was Mega humic acid followed by Cytojeep. Obtained results are in accordance with those reported by El Nagar $\boldsymbol{e t}$ al. (2012) who indicated that spraying pea plants with humic acid decreased the number of days from sowing time to flowering and Ashour et al. (2016) who found that foliar application on globe artichoke plants with $\mathrm{GA}_{3}$ at 50 ppm gave the earlier heads.

As for, the interaction effect, data in Figures (1) and (2) indicate that no significant effect among soaking with Mega humic acid or spraying either Mega humic acid or Cytojeep as well as soaking plus spraying Mega humic acid or Cytojeep or Garlic extract during the two seasons.

In this respect, the most effective treatment which exhibited the lowest number of days to flowers was the spraying of Mega humic acid in the first season or soaking plus spraying with Cytojeep.

\section{4 -Flower head yield and its components:}

Data in Table (3) indicate that the different application methods had no significant effect on early, late and total yield expressed as heads weight 
Table 2. Effect of application method and safety compounds as well as their interaction on growth characteristics of globe artichoke during 2014/2015 and 2015/2016 seasons.

\begin{tabular}{|c|c|c|c|c|c|c|c|}
\hline \multirow{2}{*}{\multicolumn{2}{|c|}{ Treatment }} & \multicolumn{2}{|c|}{ Leaves number / plant } & \multicolumn{2}{|c|}{ Plant height (cm) } & \multicolumn{2}{|c|}{$\begin{array}{c}\text { Offshoots number / } \\
\text { plant }\end{array}$} \\
\hline & & $2014 / 15$ & $2015 / 16$ & $2014 / 15$ & $2015 / 16$ & $2014 / 15$ & $2015 / 16$ \\
\hline \multicolumn{2}{|c|}{ Soaking } & 32.36 & 33.79 & 84.19 & 83.55 & 1.35 & 1.94 \\
\hline \multicolumn{2}{|c|}{ Spraying } & 32.38 & 33.01 & 85.79 & 82.23 & 1.38 & 1.78 \\
\hline \multicolumn{2}{|c|}{ So. + Sp. } & 32.85 & 33.80 & 87.72 & 89.23 & 1.59 & 2.00 \\
\hline \multicolumn{2}{|c|}{ L.S.D. at 0.05} & N.S. & N.S. & 1.60 & 2.73 & 0.20 & 0.26 \\
\hline \multicolumn{2}{|c|}{ Amino power } & 34.72 & 35.54 & 86.26 & 86.12 & 1.68 & 1.98 \\
\hline \multicolumn{2}{|c|}{ Fruiting } & 29.42 & 28.94 & 83.43 & 80.71 & 1.06 & 1.60 \\
\hline \multicolumn{2}{|c|}{ Mega humic acid } & 40.47 & 40.50 & 97.76 & 95.63 & 2.08 & 2.19 \\
\hline \multicolumn{2}{|c|}{ Chitosan } & 24.37 & 26.80 & 77.44 & 79.24 & 0.95 & 1.93 \\
\hline \multicolumn{2}{|c|}{ Garlic extract } & 32.95 & 35.84 & 89.34 & 94.08 & 1.27 & 2.32 \\
\hline \multicolumn{2}{|c|}{ Cytojeep } & 39.80 & 38.73 & 96.36 & 90.59 & 2.06 & 1.67 \\
\hline \multicolumn{2}{|c|}{ Control } & 25.98 & 28.38 & 70.71 & 68.66 & 1.00 & 1.76 \\
\hline \multicolumn{2}{|c|}{ L.S.D. at 0.05} & 1.44 & 2.59 & 2.35 & 3.24 & $\mathbf{0 . 1 7}$ & 0.26 \\
\hline \multirow{7}{*}{ 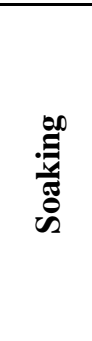 } & Amino power & 34.95 & 37.20 & 85.76 & 85.43 & 1.58 & 2.12 \\
\hline & Fruiting & 29.07 & 27.93 & 82.73 & 78.78 & 1.06 & 1.53 \\
\hline & $\begin{array}{l}\text { Mega humic } \\
\text { acid }\end{array}$ & 39.68 & 40.85 & 92.10 & 93.56 & 2.03 & 2.06 \\
\hline & Chitosan & 24.13 & 27.32 & 78.72 & 79.43 & $\mathbf{0 . 7 8}$ & 2.17 \\
\hline & Garlic extract & 32.18 & 34.81 & 88.50 & 93.89 & 1.17 & 2.57 \\
\hline & Cytojeep & 41.12 & 39.95 & 91.84 & 87.29 & 1.89 & 1.45 \\
\hline & Control & 25.37 & 28.50 & 69.71 & 66.49 & 0.96 & 1.69 \\
\hline \multirow{7}{*}{ } & Amino power & 34.57 & 33.70 & 85.83 & 83.54 & 1.59 & 1.81 \\
\hline & Fruiting & 29.18 & 29.31 & 84.78 & 79.63 & 1.11 & 1.58 \\
\hline & $\begin{array}{l}\text { Mega humic } \\
\text { acid }\end{array}$ & 40.46 & 40.01 & 97.13 & 92.28 & 1.91 & 2.17 \\
\hline & Chitosan & 25.13 & 28.00 & 75.55 & 75.91 & 0.94 & 1.56 \\
\hline & Garlic extract & 33.58 & 36.15 & 88.69 & 91.73 & 1.19 & 2.12 \\
\hline & Cytojeep & 38.51 & 36.50 & 99.63 & 87.73 & 1.98 & 1.54 \\
\hline & Control & 25.20 & 28.40 & 68.97 & 64.84 & 0.98 & 1.72 \\
\hline \multirow{7}{*}{$\begin{array}{l}\dot{2} \\
+ \\
\dot{0}\end{array}$} & Amino power & 34.66 & 35.72 & 87.22 & 89.40 & 1.88 & 2.02 \\
\hline & Fruiting & 30.02 & 29.57 & 82.79 & 83.73 & 1.01 & 1.70 \\
\hline & $\begin{array}{l}\text { Mega humic } \\
\text { acid }\end{array}$ & 41.26 & 40.63 & 104.07 & 101.06 & 2.29 & 2.36 \\
\hline & Chitosan & 23.85 & 25.10 & 78.07 & 82.40 & 1.15 & 1.75 \\
\hline & Garlic extract & 33.07 & 36.57 & 90.83 & 96.62 & 1.45 & 2.29 \\
\hline & Cytojeep & 39.76 & 40.76 & 97.62 & 96.75 & 2.30 & 2.01 \\
\hline & Control & 27.37 & 28.25 & 73.46 & 74.66 & 1.07 & 1.87 \\
\hline \multicolumn{2}{|c|}{ L.S.D. at 0.05} & 2.57 & 4.24 & 4.09 & 5.85 & 0.50 & 0.50 \\
\hline
\end{tabular}

So. = soaking, Sp. = spraying, L.S.D. = low significant different. 

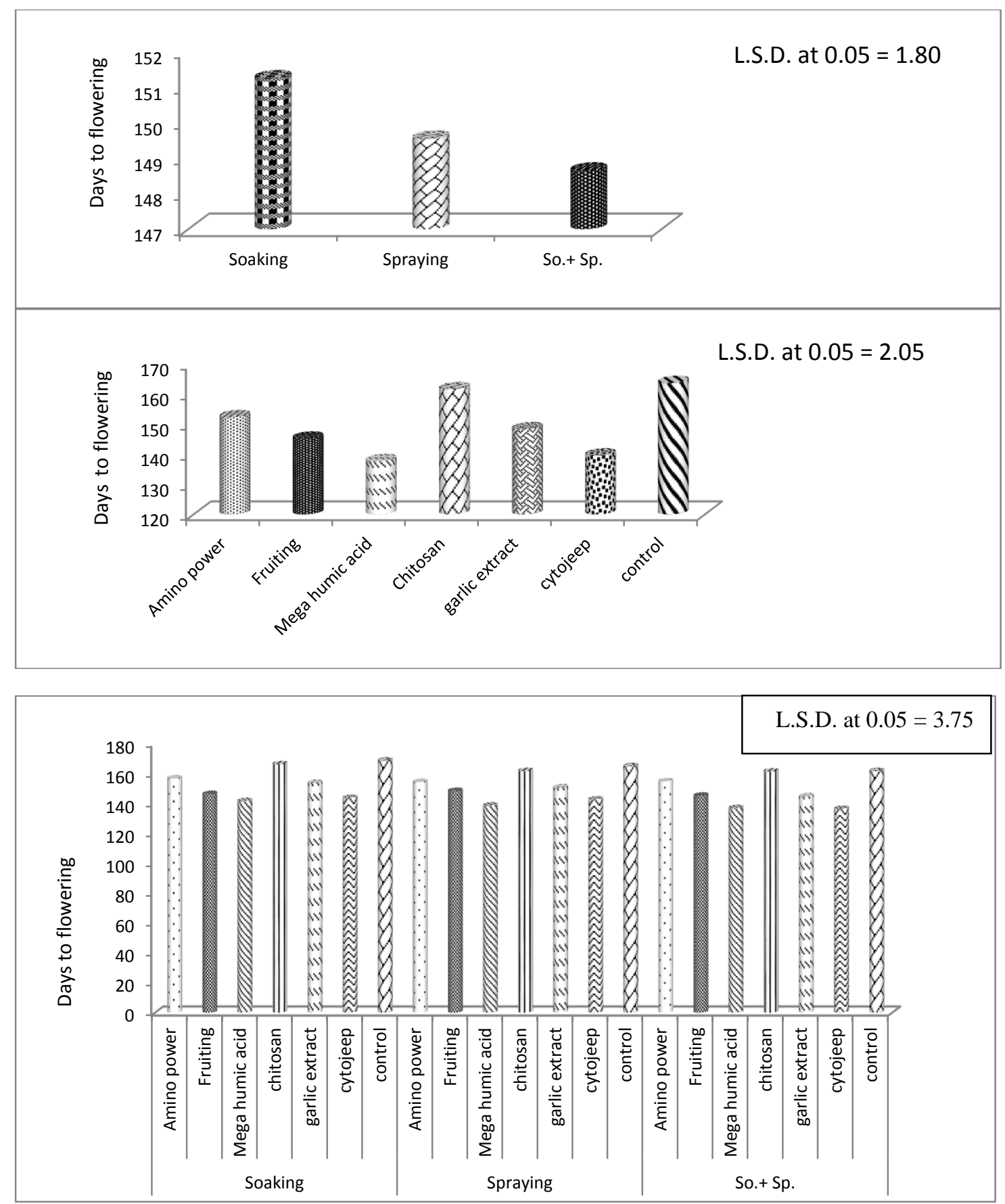

Fig. 1. Effect of application method and safety compounds as well as their interaction on flowering behavior of globe artichoke during 2014/2015 season. 


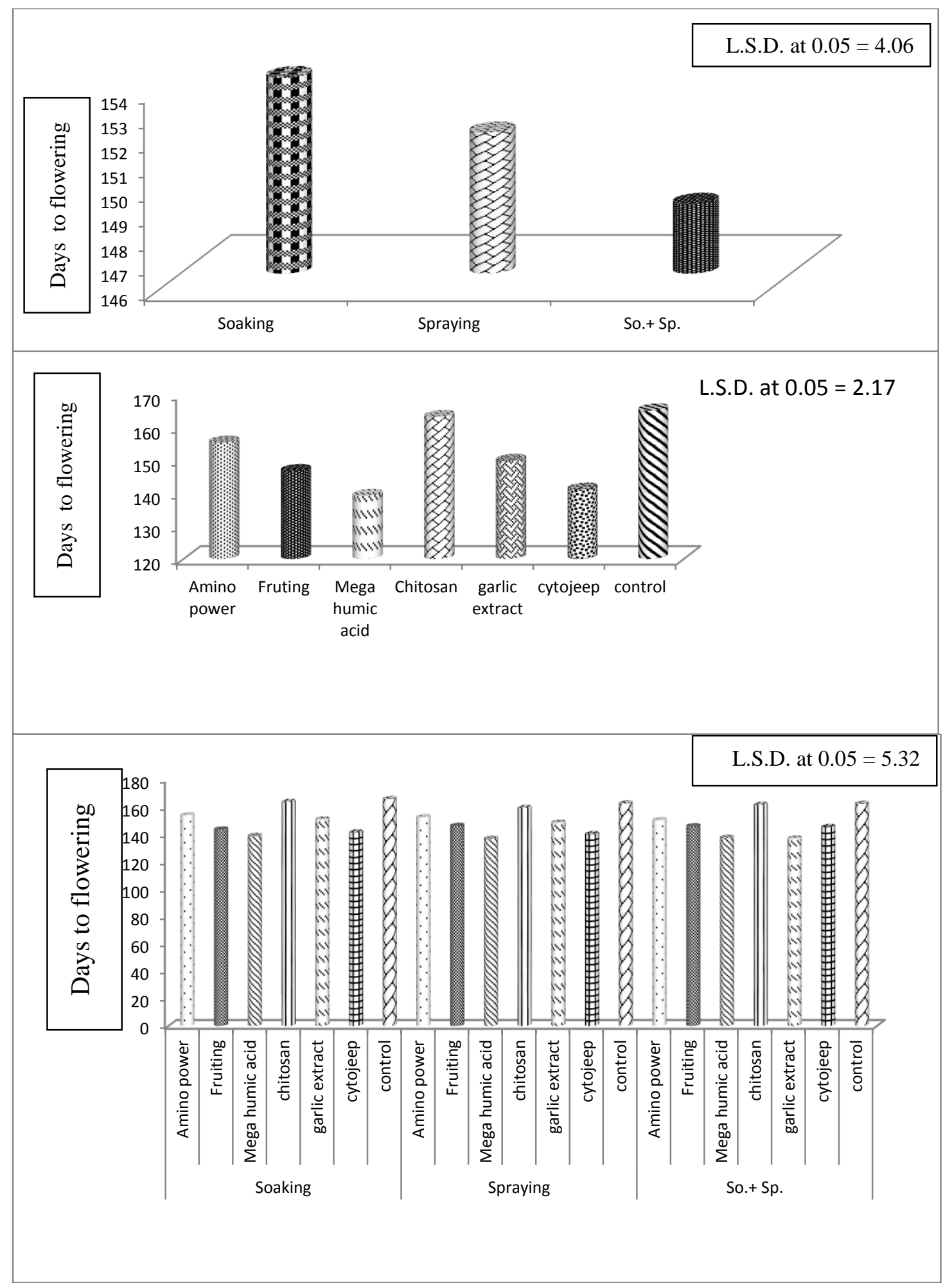

Fig. 2. Effect of application method and safety compounds as well as their interaction on flowering behavior of globe artichoke during 2015/2016 season. 
Table 3. Effect of application method and safety compounds as well as their interaction on flower head weight per plant $(\mathrm{kg})$ of globe artichoke during 2014/2015 and 2015/2016 seasons

\begin{tabular}{|c|c|c|c|c|c|c|c|}
\hline \multirow{2}{*}{\multicolumn{2}{|c|}{ Treatment }} & \multicolumn{2}{|c|}{ Early yield } & \multicolumn{2}{|c|}{ Late yield } & \multicolumn{2}{|c|}{ Total yield } \\
\hline & & $2014 / 15$ & $2015 / 16$ & $2014 / 15$ & $2015 / 16$ & $2014 / 15$ & $2015 / 16$ \\
\hline \multicolumn{2}{|c|}{ Soaking } & 1.06 & 1.60 & 5.15 & 7.42 & 6.22 & 9.02 \\
\hline \multicolumn{2}{|c|}{ Spraying } & 1.15 & 1.68 & 5.59 & 7.91 & 6.75 & 9.60 \\
\hline \multicolumn{2}{|c|}{ So. + Sp. } & 1.20 & 1.73 & 5.67 & 8.07 & 6.87 & 9.81 \\
\hline \multicolumn{2}{|c|}{ L.S.D. at 0.05} & 0.02 & N.S. & 0.19 & N.S. & 0.20 & N.S. \\
\hline \multicolumn{2}{|c|}{ Amino power } & 0.97 & 1.53 & 4.56 & 6.80 & 5.53 & 8.33 \\
\hline \multicolumn{2}{|c|}{ Fruiting } & 1.42 & 1.97 & 7.19 & 9.64 & 8.61 & 11.62 \\
\hline \multicolumn{2}{|c|}{ Mega humic acid } & 1.63 & 2.17 & 7.77 & 10.34 & 9.40 & 12.51 \\
\hline \multicolumn{2}{|c|}{ Chitosan } & 0.86 & 1.38 & 3.80 & 5.91 & 4.67 & 7.29 \\
\hline \multicolumn{2}{|c|}{ Garlic extract } & 1.23 & 1.82 & 6.33 & 8.75 & 7.56 & 10.58 \\
\hline \multicolumn{2}{|c|}{ Cytojeep } & 1.07 & 1.61 & 5.54 & 7.83 & 6.62 & 9.45 \\
\hline \multicolumn{2}{|c|}{ Control } & 0.79 & 1.22 & 3.10 & 5.32 & 3.90 & 6.54 \\
\hline \multicolumn{2}{|c|}{ L.S.D. at 0.05} & 0.29 & 0.19 & 0.15 & $\mathbf{0 . 1 0}$ & 0.62 & 1.28 \\
\hline \multirow{7}{*}{ 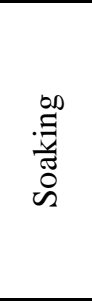 } & Amino power & 0.89 & 1.49 & 4.20 & 6.40 & 5.67 & 7.89 \\
\hline & Fruiting & 1.34 & 1.91 & 6.72 & 9.15 & 8.07 & 11.06 \\
\hline & Mega humic acid & 1.54 & 2.05 & 7.37 & 9.76 & 8.92 & 11.81 \\
\hline & Chitosan & 0.78 & 1.31 & 3.53 & 5.66 & 4.31 & 6.98 \\
\hline & Garlic extract & 1.12 & 1.79 & 5.87 & 8.23 & 7.00 & 10.03 \\
\hline & Cytojeep & 0.98 & 1.44 & 5.22 & 7.52 & 6.21 & 8.97 \\
\hline & Control & 0.77 & 1.20 & 3.15 & 5.20 & 3.92 & 6.40 \\
\hline \multirow{7}{*}{ 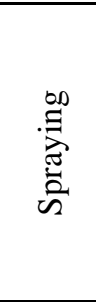 } & Amino power & 1.00 & 1.54 & 4.66 & 6.94 & 5.67 & 8.48 \\
\hline & Fruiting & 1.44 & 2.00 & 7.34 & 9.81 & 8.87 & 11.82 \\
\hline & Mega humic acid & 1.63 & 2.20 & 7.94 & 10.58 & 9.57 & 12.79 \\
\hline & Chitosan & 0.89 & 1.39 & 3.88 & 6.02 & 4.77 & 7.42 \\
\hline & Garlic extract & 1.25 & 1.80 & 6.48 & 8.90 & 7.74 & 10.70 \\
\hline & Cytojeep & 1.09 & 1.68 & 5.65 & 7.83 & 6.74 & 9.52 \\
\hline & Control & 0.78 & 1.17 & 3.20 & 5.28 & 3.99 & 6.45 \\
\hline \multirow{7}{*}{$\begin{array}{l}\dot{2} \\
\dot{n} \\
\dot{\Leftrightarrow} \\
\dot{\Leftrightarrow}\end{array}$} & Amino power & 1.01 & 1.56 & 4.82 & 7.05 & 5.84 & 8.62 \\
\hline & Fruiting & 1.48 & 2.01 & 7.51 & 9.97 & 8.99 & 11.99 \\
\hline & Mega humic acid & 1.71 & 2.26 & 8.01 & 10.67 & 9.72 & 12.94 \\
\hline & Chitosan & 0.91 & 1.43 & 4.01 & 6.05 & 4.93 & 7.49 \\
\hline & Garlic extract & 1.31 & 1.87 & 6.63 & 9.13 & 7.95 & 11.01 \\
\hline & Cytojeep & 1.14 & 1.71 & 5.76 & 8.14 & 6.90 & 9.86 \\
\hline & Control & 0.83 & 1.27 & 2.95 & 5.47 & 3.79 & 6.75 \\
\hline \multicolumn{2}{|c|}{ L.S.D. at 0.05} & 0.52 & 0.27 & 0.31 & 0.21 & 0.32 & 0.25 \\
\hline
\end{tabular}

\section{So. = Soaking , Sp. = spraying , L.S.D. = low significant different.}

per plant in the second season. Meanwhile, in the first season, using soaking plus spraying increased significantly early yield.

As for, late and total yield, data show that there were no significant effects among spraying and soaking plus spraying. However, using soaking plus spraying increased significantly late yield compared with soaking only.

In this respect, data in the same Table show that using Mega humic acid increased significantly early, late and total yield expressed as heads weight per plant. These results agree with those obtained by Kandil (2014) who showed that yield of pea increased significantly by using humic acid and Avinash $\boldsymbol{e t}$ al. (2017) who indicated that using humic acid to treated capsicum plants increased significantly yield per plant.
Concerning the interaction effect, data in the same Table indicate that spraying only or soaking plus spraying with Mega humic acid increased significantly early, late and total yield as heads weight per plant except early yield in the second season. There was no significant effect when compared with soaking only of Mega humic acid or spraying as well as soaking plus spraying of Fruiting.

\section{5 - Head characteristics}

Data in Table (4) illustrate that soaking plus spraying reflected the highest head weight during the first season of early yield and the second season of the late yield with significant difference compared with soaking treatment only while there was no significant effect when compared with spraying treatment. Referring to the effect of safety compounds, using Mega humic acid or Fruiting exhibited the highest head weight with significant differences as compared 
with the other treatments in either early or late yield in the two seasons except late yield in the second season. Avinash et al. (2017) showed that using humic acid to treated capsicum plants showed significant differences in fruit weight.

Also, El-Zohiri (2015) found that maximum increaseing head weight was obtained due to treating globe artichoke plants with $\mathrm{GA}_{3}$. Regarding the effect of their interaction, data presented in the same Table reveal that soaking plus spraying of Mega humic acid increased average head weight in both of early and late yield during both seasons with significant differences except Fruiting when treating as spraying or soaking plus spraying during the second season on early yield. As well as using Mega

Table 4. Effect of application method and safety compounds as well as their interaction on flower head physical quality (average head weight and edible part weight) of globe artichoke during 2014/2015 and 2015/2016 seasons

\begin{tabular}{|c|c|c|c|c|c|c|c|c|c|}
\hline \multirow{2}{*}{\multicolumn{2}{|c|}{ Treatment }} & \multicolumn{2}{|c|}{$\begin{array}{l}\text { Average head } \\
\text { weight (g) } \\
\text { (Early yield) }\end{array}$} & \multicolumn{2}{|c|}{$\begin{array}{c}\text { Average head } \\
\text { weight (g) } \\
\text { (Late yield) }\end{array}$} & \multicolumn{2}{|c|}{$\begin{array}{c}\text { Edible part } \\
\text { weight (g) } \\
\text { (Early yield) }\end{array}$} & \multicolumn{2}{|c|}{$\begin{array}{c}\text { Edible part } \\
\text { weight (g) } \\
\text { (Late yield) }\end{array}$} \\
\hline & & $2014 / 15$ & $2015 / 16$ & $2014 / 15$ & $2015 / 16$ & $2014 / 15$ & $2015 / 16$ & $2014 / 15$ & $2015 / 16$ \\
\hline \multicolumn{2}{|c|}{ Soaking } & 271.00 & 279.45 & 229.50 & 237.89 & 70.39 & 78.63 & 59.64 & 67.99 \\
\hline \multicolumn{2}{|c|}{ Spraying } & 272.51 & 280.75 & 231.72 & 240.04 & 71.18 & 79.46 & 60.75 & 69.60 \\
\hline \multicolumn{2}{|c|}{ So.+ Sp. } & 273.10 & 281.90 & 230.38 & 242.84 & 71.25 & 79.43 & 61.30 & 70.38 \\
\hline \multicolumn{2}{|c|}{ L.S.D. at 0.05} & 1.64 & N.S. & N.S. & 3.82 & N.S. & N.S. & N.S. & N.S. \\
\hline \multicolumn{2}{|c|}{ Amino power } & 268.01 & 275.84 & 224.62 & 233.19 & 66.77 & 72.92 & 59.19 & 68.31 \\
\hline \multicolumn{2}{|c|}{ Fruiting } & 281.09 & 290.24 & 246.60 & 255.55 & 78.05 & 86.15 & 66.76 & 75.87 \\
\hline \multicolumn{2}{|c|}{ Mega humic acid } & 281.76 & 290.22 & 252.09 & 259.57 & 84.63 & 93.26 & 69.43 & 77.77 \\
\hline \multicolumn{2}{|c|}{ Chitosan } & 265.34 & 273.90 & 215.70 & 224.14 & 64.19 & 72.70 & 52.21 & 60.66 \\
\hline \multicolumn{2}{|c|}{ Garlic extract } & 275.34 & 283.79 & 240.29 & 248.86 & 67.60 & 76.19 & 61.55 & 70.13 \\
\hline \multicolumn{2}{|c|}{ Cytojeep } & 270.60 & 279.03 & 232.51 & 241.07 & 71.54 & 80.46 & 64.29 & 72.76 \\
\hline \multicolumn{2}{|c|}{ Control } & 263.25 & 271.85 & 201.92 & 219.43 & 63.78 & 72.55 & 50.54 & 59.76 \\
\hline \multicolumn{2}{|c|}{ L.S.D. at 0.05} & 2.738 & 3.08 & 9.54 & 3.15 & 2.28 & 3.99 & 2.13 & 2.11 \\
\hline \multirow{7}{*}{ 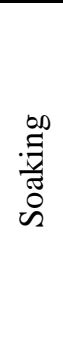 } & Amino power & 267.28 & 275.88 & 223.53 & 232.06 & 66.83 & 73.51 & 58.56 & 67.03 \\
\hline & Fruiting & 279.78 & 288.19 & 242.51 & 252.17 & 76.91 & 85.25 & 64.96 & 73.53 \\
\hline & $\begin{array}{l}\text { Mega humic } \\
\text { acid }\end{array}$ & 279.25 & 287.79 & 249.56 & 256.40 & 84.47 & 91.83 & 68.03 & 76.34 \\
\hline & Chitosan & 262.63 & 271.22 & 214.22 & 222.75 & 64.68 & 73.00 & 52.34 & 60.84 \\
\hline & Garlic extract & 273.79 & 282.22 & 236.26 & 244.79 & 66.71 & 75.31 & 60.81 & 67.84 \\
\hline & Cytojeep & 270.09 & 278.21 & 231.16 & 239.45 & 69.28 & 79.07 & 62.00 & 70.64 \\
\hline & Control & 264.16 & 272.63 & 209.25 & 217.63 & 63.85 & 72.46 & 50.78 & 59.31 \\
\hline \multirow{7}{*}{ 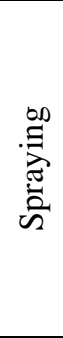 } & Amino power & 269.22 & 275.25 & 223.22 & 231.76 & 68.06 & 74.22 & 59.16 & 67.53 \\
\hline & Fruiting & 281.91 & 290.38 & 248.08 & 256.70 & 77.66 & 85.78 & 66.36 & 76.59 \\
\hline & $\begin{array}{l}\text { Mega humic } \\
\text { acid }\end{array}$ & 279.31 & 287.88 & 252.02 & 259.19 & 84.75 & 93.44 & 69.47 & 77.88 \\
\hline & Chitosan & 267.41 & 276.09 & 214.88 & 223.16 & 64.46 & 73.16 & 51.63 & 60.09 \\
\hline & Garlic extract & 275.13 & 283.60 & 241.38 & 249.98 & 67.13 & 75.75 & 62.31 & 70.63 \\
\hline & Cytojeep & 272.38 & 281.03 & 233.11 & 241.44 & 71.84 & 80.40 & 65.03 & 73.50 \\
\hline & Control & 262.19 & 271.00 & 209.36 & 218.04 & 64.35 & 73.45 & 51.32 & 61.01 \\
\hline \multirow{7}{*}{$\begin{array}{l}\dot{2} \\
\dot{n} \\
\dot{0}\end{array}$} & Amino power & 267.54 & 276.41 & 227.13 & 235.74 & 65.43 & 71.03 & 59.84 & 70.38 \\
\hline & Fruiting & 281.59 & 292.16 & 249.22 & 275.78 & 79.58 & 87.41 & 68.97 & 77.51 \\
\hline & $\begin{array}{l}\text { Mega humic } \\
\text { acid }\end{array}$ & 286.72 & 295.00 & 254.70 & 263.10 & 84.66 & 94.50 & 70.78 & 79.10 \\
\hline & Chitosan & 266.00 & 274.38 & 218.00 & 226.50 & 63.43 & 71.94 & 52.66 & 61.06 \\
\hline & Garlic extract & 277.09 & 285.65 & 243.23 & 251.81 & 68.97 & 77.51 & 61.51 & 71.51 \\
\hline & Cytojeep & 269.34 & 277.85 & 233.25 & 242.32 & 73.51 & 81.91 & 65.83 & 74.14 \\
\hline & Control & 263.41 & 271.94 & 187.17 & 222.63 & 63.16 & 71.73 & 49.53 & 58.97 \\
\hline \multicolumn{2}{|c|}{ L.S.D. at 0.05} & 4.68 & 5.82 & 17.74 & 6.31 & 4.32 & 0.93 & 3.89 & 4.79 \\
\hline
\end{tabular}

So. = Soaking , Sp. = spraying , L.S.D. = low significant different

humic acid or Fruiting as treated by soaking or spraying in addition to Garlic extract as spraying or soaking plus spraying during the first season of late yield meanwhile, using Mega humic acid as spraying 
or Fruiting as soaking plus spraying during second season of late yield.

Concerning the edible part weight, data presented in Table (4) reveal that the application method had no significant effect on edible part weight in early and late yield during both seasons. Moreover, data indicate that using Mega humic acid increased significant edible part weight in both early and late yield except late yield in the second season when compared with Fruiting.

Regarding the effect of interaction, data in the same Table indicate that using Mega humic acid when treated in various application methods increased significantly edible part weight in early yield during both seasons, while using Mega humic acid in all different application methods increased edible part weight in late yield with no significant difference during both seasons.

\section{6 - Head chemical composition}

Concerning the data in Tables (5) and (6) there were no significant differences among application method for dry matter. As for, using soaking only increased significantly inuline percentage as compared with the other treatments except for during late yield there were no significant differences between soaking and spraying in both seasons. While, using soaking plus spraying as application method decreased significantly fibers content as compared with soaking only but the decreasing not significant as compared with spraying during early and late yield in both seasons. While, soaking plus spraying increased total phenolic compounds during the first season of early yield and decreased it during the first season of late yield but there were no significant difference among the different application methods on total phenolic compounds during the second season in both early and late yield.

Regarding the effect of safety compounds, data in the same Tables indicate that using Mega humic acid increased dry matter percentage in either early or late yield during both seasons. These results confirmed those obtained by Mohsen et al. (2017) who noted that garlic plants treated with humic acid increasing bulb dry matter. While, using Fruiting increased inuline percentage in early yield during both seasons also in late yield or using Amino power in two seasons. These results disagree with those obtained by El-Zohiri (2015) who indicated that $\mathrm{GA}_{3}$ application gave the lowest inuline content.

As for fibers content and total phenolic compounds, data in Table (6) show that using amino power decreased fiber content in early yield in both seasons.But in the late yield the lowest fibers content was obtained by the using of Mega humic acid in both seasons meanwhile using Mega humic acid decreased total phenolic compounds but the lowest total phenols due to using garlic extract in late yield.

Regarding the effect of interaction, data in the same Table reveal that soaking plus spraying with Fruiting increased dry matter in early yield during first season while, soaking plus spraying or spraying only with Mega humic acid reflected the highest values of dry matter during second season as well as late yield in both seasons.

Moreover, spraying Amino power or Fruiting increased significantly inuline percentage during early yield in both seasons while, spraying Amino power or Fruiting as well as soaking in Garlic extract increased significantly inuline percentage during late yield during both seasons.

Moreover, the lowest fibers content obtained from soaking followed by spraying with Amino power or Cytojeep in early yield during the first and the second season respectively, while in late yield, spraying Fruiting or Mega humic acid decreased fibers content in the first and the second season respectively . Meanwhile, soaking plus spraying of in Mega humic acid decreased total phenols in early yield but spraying only with Cytojeep or Garlic extract decreased total phenols in late yield. 
Table 5. Effect of application method and safety compounds as well as their interaction on dry matter and inuline percentage of globe artichoke during 2014/2015 and 2015/2016 seasons

\begin{tabular}{|c|c|c|c|c|c|c|c|c|c|}
\hline \multirow{2}{*}{\multicolumn{2}{|c|}{ Treatment }} & \multicolumn{2}{|c|}{$\begin{array}{l}\text { Dry matter (\%) } \\
\text { early yield }\end{array}$} & \multicolumn{2}{|c|}{$\begin{array}{c}\text { Dry matter (\%) } \\
\text { late yield }\end{array}$} & \multicolumn{2}{|c|}{$\begin{array}{c}\text { Inuline }(\%) \\
\text { early yield }\end{array}$} & \multicolumn{2}{|c|}{$\begin{array}{c}\text { Inuline (\%) } \\
\text { late yield }\end{array}$} \\
\hline & & $2014 / 15$ & $2015 / 16$ & $2014 / 15$ & $2015 / 16$ & $2014 / 15$ & $2015 / 16$ & $2014 / 15$ & $2015 / 16$ \\
\hline \multicolumn{2}{|c|}{ Soaking } & 10.32 & 10.18 & 14.13 & 14.04 & 0.39 & 0.40 & 0.25 & 0.25 \\
\hline \multicolumn{2}{|c|}{ Spraying } & 10.26 & 10.23 & 14.28 & 14.43 & 0.38 & 0.38 & 0.25 & 0.25 \\
\hline \multicolumn{2}{|c|}{ So.+ Sp. } & 10.40 & 10.53 & 14.43 & 14.60 & 0.36 & 0.37 & 0.24 & 0.24 \\
\hline \multicolumn{2}{|c|}{ L.S.D. at 0.05} & N.S. & N.S. & N.S. & N.S. & 0.003 & 0.004 & 0.006 & 0.008 \\
\hline \multicolumn{2}{|c|}{ Amino power } & 10.03 & 9.99 & 14.05 & 13.91 & 0.39 & 0.39 & 0.25 & 0.26 \\
\hline \multicolumn{2}{|c|}{ Fruiting } & 10.83 & 10.60 & 14.95 & 14.52 & 0.43 & 0.43 & 0.25 & 0.26 \\
\hline \multicolumn{2}{|c|}{ Mega humic acid } & 11.09 & 10.88 & 15.04 & 14.94 & 0.36 & 0.36 & 0.24 & 0.25 \\
\hline \multicolumn{2}{|c|}{ Chitosan } & 9.79 & 10.01 & 13.75 & 14.06 & 0.39 & 0.39 & 0.24 & 0.24 \\
\hline \multicolumn{2}{|c|}{ Garlic extract } & 10.47 & 10.48 & 14.16 & 14.72 & 0.35 & 0.35 & 0.24 & 0.25 \\
\hline \multicolumn{2}{|c|}{ Cytojeep } & 10.30 & 10.24 & 14.48 & 14.29 & 0.35 & 0.35 & 0.24 & 0.24 \\
\hline \multicolumn{2}{|c|}{ Control } & 9.78 & 9.99 & 13.54 & 14.08 & 0.38 & 0.38 & 0.24 & 0.24 \\
\hline \multicolumn{2}{|c|}{ L.S.D. at 0.05} & 0.48 & 0.39 & 0.46 & 0.73 & 0.006 & 0.005 & 0.004 & 0.005 \\
\hline \multirow{7}{*}{ 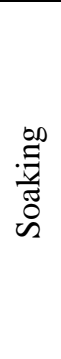 } & Amino power & 10.05 & 9.85 & 14.12 & 13.76 & 0.31 & 0.31 & 0.23 & 0.23 \\
\hline & Fruiting & 10.74 & 10.40 & 14.68 & 13.99 & 0.33 & 0.33 & 0.25 & 0.25 \\
\hline & Mega humic acid & 11.00 & 10.98 & 14.86 & 14.64 & 0.32 & 0.32 & 0.25 & 0.25 \\
\hline & Chitosan & 9.76 & 9.67 & 13.50 & 13.98 & 0.41 & 0.41 & 0.24 & 0.24 \\
\hline & Garlic extract & 10.59 & 10.25 & 14.09 & 14.28 & 0.44 & 0.44 & 0.28 & 0.29 \\
\hline & Cytojeep & 10.35 & 10.13 & 14.26 & 13.91 & 0.43 & 0.43 & 0.27 & 0.27 \\
\hline & Control & 9.74 & 9.96 & 13.42 & 13.74 & 0.51 & 0.51 & 0.24 & 0.24 \\
\hline \multirow{7}{*}{ 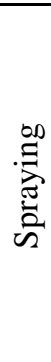 } & Amino power & 9.93 & 10.00 & 13.94 & 13.77 & 0.55 & 0.55 & 0.28 & 0.28 \\
\hline & Fruiting & 10.51 & 10.45 & 15.22 & 14.70 & 0.55 & 0.55 & 0.28 & 0.28 \\
\hline & Mega humic acid & 11.15 & 10.65 & 15.24 & 15.24 & 0.29 & 0.29 & 0.22 & 0.23 \\
\hline & Chitosan & 9.84 & 10.14 & 13.96 & 13.75 & 0.31 & 0.31 & 0.23 & 0.23 \\
\hline & Garlic extract & 10.42 & 10.41 & 13.98 & 14.71 & 0.31 & 0.31 & 0.23 & 0.24 \\
\hline & Cytojeep & 10.14 & 10.34 & 14.22 & 14.39 & 0.31 & 0.31 & 0.23 & 0.24 \\
\hline & Control & 9.83 & 9.64 & 13.43 & 14.41 & 0.32 & 0.33 & 0.25 & 0.26 \\
\hline \multirow{7}{*}{$\begin{array}{l}\dot{\theta} \\
+ \\
\dot{0}\end{array}$} & Amino power & 10.11 & 10.14 & 14.08 & 14.19 & 0.32 & 0.32 & 0.25 & 0.26 \\
\hline & Fruiting & 11.25 & 10.95 & 14.93 & 14.86 & 0.41 & 0.41 & 0.23 & 0.24 \\
\hline & Mega humic acid & 11.12 & 11.02 & 15.04 & 14.94 & 0.45 & 0.45 & 0.26 & 0.27 \\
\hline & Chitosan & 9.77 & 10.23 & 13.80 & 14.45 & 0.44 & 0.44 & 0.26 & 0.26 \\
\hline & Garlic extract & 10.40 & 10.76 & 14.41 & 15.15 & 0.29 & 0.29 & 0.22 & 0.22 \\
\hline & Cytojeep & 10.41 & 10.25 & 14.95 & 14.55 & 0.30 & 0.30 & 0.22 & 0.22 \\
\hline & Control & 9.77 & 10.38 & 13.78 & 14.08 & 0.31 & 0.31 & 0.22 & 0.22 \\
\hline \multicolumn{2}{|c|}{ L.S.D. at 0.05} & 0.84 & 0.55 & 0.93 & 0.55 & 0.001 & 0.004 & 0.009 & 0.008 \\
\hline
\end{tabular}

So. = soaking , Sp. = spraying , L.S.D. = low significant different 
Table 6. Effect of application method and safety compounds as well as their interaction on fibers content and total phenolic of globe artichoke during 2014/2015 and 2015/2016 seasons.

\begin{tabular}{|c|c|c|c|c|c|c|c|c|c|}
\hline \multirow{2}{*}{\multicolumn{2}{|c|}{ Treatment }} & \multicolumn{2}{|c|}{$\begin{array}{c}\text { Fibers content } \\
\text { (mg/g D.W.) } \\
\text { early yield }\end{array}$} & \multicolumn{2}{|c|}{$\begin{array}{c}\text { Fibers content } \\
\text { (mg/g D.W.) } \\
\text { late yield } \\
\end{array}$} & \multicolumn{2}{|c|}{$\begin{array}{c}\text { Total phenolic } \\
\text { compounds }(\%) \\
\text { early yield } \\
\end{array}$} & \multicolumn{2}{|c|}{$\begin{array}{c}\text { Total phenolic } \\
\text { Compounds }(\%) \\
\text { late yield } \\
\end{array}$} \\
\hline & & $2014 / 15$ & $2015 / 16$ & $2014 / 15$ & $2015 / 16$ & $2014 / 15$ & $2015 / 16$ & $2014 / 15$ & $2015 / 16$ \\
\hline \multicolumn{2}{|c|}{ Soaking } & 5.77 & 5.71 & 7.58 & 7.52 & 2.01 & 2.04 & 2.22 & 2.26 \\
\hline \multicolumn{2}{|c|}{ Spraying } & 5.11 & 5.07 & 6.90 & 6.76 & 2.01 & 2.04 & 2.20 & 2.24 \\
\hline \multicolumn{2}{|c|}{ So.+ Sp. } & 5.05 & 5.07 & 6.87 & 7.01 & 2.05 & 2.03 & 2.19 & 2.23 \\
\hline \multicolumn{2}{|c|}{ L.S.D. at 0.05} & 0.30 & 0.39 & 0.26 & 0.48 & 0.02 & N.S. & 0.02 & 0.02 \\
\hline \multicolumn{2}{|c|}{ Amino power } & 4.78 & 4.89 & 7.14 & 6.81 & 1.90 & 1.90 & 2.07 & 2.11 \\
\hline \multicolumn{2}{|c|}{ Fruiting } & 5.14 & 5.28 & 6.43 & 6.54 & 2.02 & 2.03 & 2.31 & 2.35 \\
\hline \multicolumn{2}{|c|}{ Mega humic acid } & 5.07 & 5.03 & 6.38 & 6.49 & 1.74 & 1.75 & 2.03 & 2.07 \\
\hline \multicolumn{2}{|c|}{ Chitosan } & 5.76 & 5.94 & 7.90 & 7.75 & 2.06 & 2.05 & 2.37 & 2.41 \\
\hline \multicolumn{2}{|c|}{ garlic extract } & 5.34 & 5.08 & 7.29 & 7.62 & 1.78 & 1.80 & 1.91 & 1.95 \\
\hline \multicolumn{2}{|c|}{ Cytojeep } & 5.08 & 4.93 & 6.82 & 6.99 & 2.30 & 2.27 & 2.31 & 2.36 \\
\hline \multicolumn{2}{|c|}{ Control } & 5.99 & 5.85 & 7.85 & 7.50 & 2.37 & 2.47 & 2.42 & 2.46 \\
\hline \multicolumn{2}{|c|}{ L.S.D. at 0.05} & 0.26 & $\mathbf{0 . 3 3}$ & 0.23 & 0.41 & 0.06 & 0.06 & 0.04 & 0.04 \\
\hline \multirow{7}{*}{ 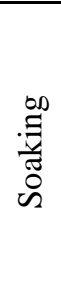 } & Amino power & 5.47 & 5.37 & 7.67 & 7.63 & 1.89 & 1.93 & 2.04 & 2.08 \\
\hline & Fruiting & 5.90 & 6.03 & 7.05 & 6.97 & 2.00 & 2.00 & 2.42 & 2.45 \\
\hline & Mega humic acid & 5.86 & 5.75 & 6.93 & 7.32 & 1.77 & 1.74 & 2.07 & 2.11 \\
\hline & Chitosan & 5.92 & 5.55 & 8.07 & 7.95 & 2.06 & 2.00 & 2.33 & 2.36 \\
\hline & garlic extract & 5.73 & 5.43 & 7.93 & 8.04 & 1.76 & 1.80 & 1.90 & 1.93 \\
\hline & Cytojeep & 5.68 & 5.75 & 7.26 & 7.08 & 2.19 & 2.34 & 2.42 & 2.45 \\
\hline & Control & 5.86 & 6.10 & 8.17 & 7.68 & 2.41 & 2.51 & 2.37 & 2.41 \\
\hline \multirow{7}{*}{ 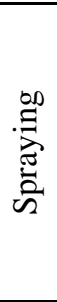 } & Amino power & 4.45 & 4.54 & 6.86 & 6.11 & 1.86 & 1.90 & 2.07 & 2.11 \\
\hline & Fruiting & 4.76 & 4.78 & 5.90 & 6.13 & 2.00 & 2.06 & 2.31 & 2.35 \\
\hline & Mega humic acid & 4.45 & 4.71 & 6.12 & 6.05 & 1.76 & 1.80 & 2.02 & 2.06 \\
\hline & Chitosan & 5.80 & 6.26 & 7.78 & 7.44 & 2.05 & 2.05 & 2.38 & 2.42 \\
\hline & garlic extract & 5.21 & 4.83 & 6.84 & 7.00 & 1.75 & 1.75 & 2.31 & 1.91 \\
\hline & Cytojeep & 4.85 & 4.70 & 6.80 & 7.04 & 2.31 & 2.26 & 1.87 & 2.38 \\
\hline & Control & 6.24 & 5.70 & 7.78 & 7.59 & 2.37 & 2.47 & 2.41 & 2.46 \\
\hline \multirow{7}{*}{$\begin{array}{l}\dot{2} \\
\dot{n} \\
\dot{\theta}\end{array}$} & Amino power & 4.40 & 4.75 & 6.88 & 6.71 & 1.95 & 1.88 & 2.09 & 2.13 \\
\hline & Fruiting & 4.77 & 5.02 & 6.34 & 6.54 & 2.05 & 2.02 & 2.19 & 2.24 \\
\hline & Mega humic acid & 4.91 & 4.64 & 6.13 & 6.09 & 1.69 & 1.73 & 2.00 & 2.04 \\
\hline & Chitosan & 5.57 & 6.01 & 7.84 & 7.86 & 2.07 & 2.10 & 2.42 & 2.47 \\
\hline & garlic extract & 5.10 & 4.98 & 7.10 & 7.84 & 1.84 & 1.86 & 1.96 & 2.01 \\
\hline & Cytojeep & 4.72 & 4.35 & 6.40 & 6.84 & 2.41 & 2.21 & 2.20 & 2.25 \\
\hline & Control & 5.87 & 5.74 & 7.60 & 7.22 & 2.32 & 2.43 & 2.48 & 2.50 \\
\hline \multicolumn{2}{|c|}{ L.S.D. at 0.05} & 0.35 & 0.70 & 0.36 & 0.56 & 0.10 & 0.10 & 0.07 & 0.08 \\
\hline
\end{tabular}

So. = soaking , Sp. = spraying , L.S.D. = low significant different

\section{Conclusion}

It could be concluded that under the experiment condition, spraying or soaking plus spraying of Mega humic acid on globe artichoke plants might be recommended for increasing the vegetative growth traits, flower head yield and its components as well as flower head quality.

\section{References}

A.O.A.C. (2000). Official Methods of Analysis, 13르, Association of Official Agriculture Chemists, Washington, D.C.
Akinremi, O.O., H.H. Janzen, R.L. Lemke and F.J. Larney (2000). Response of canola, wheat and green beans to leonardite additions. Canadian J. of Soil Sci., 80: 437-443.

Al Mayahi, Z. and H., Fayadh (2016). The effects of garlic extract, its application method and their interaction on growth and yield of potato, (Solanum tuberosum L.) Cv. Latonia. Adv.In Agric. \& Bot. Bioflux, 7(1): 59-69, 18 ref.

Avinash S.N., C.A. Srinivasamurthy, S. Bhaskar and N.B. Prakash (2017). Characterization, Extraction and Foliar Spray of Fortified Humic Acid on Quality of Capsicum. Int. J.Curr. Microbiol.App.Sci 6(10): 2265-2272, 18 ref. 
Ashour, H. M. Shadia A. Ismail, Nadia M. Ibrahem and Amany A. Abd El- Latif (2016).Impact of plant growth regulators application on early yield and productivity of globe artichoke (Cynara scolymus L.). American-Eurasian J. Agric. \& Environ. Sci., 16 (8): 1484-1497, 34 ref.

El-Adawy, A.Y. (2017) Effect of foliar spraying with some bio-stimulants on earliness and productivity of some globe artichoke varaietes. M.Sc. Thesis, Fac. Agric., Demietta Univ., 93 pp.

El Nagar, M.M., N.S.A. Shafshak, F.A. Abo Sedera, A.A. Esmail And A.S. Kamel (2012). Effect of foliar spray by some natural stimulating compounds on growth, yield and chemical composition of peas (Pisum sativum L.). Annals of Agric. Sci., Moshtohor, 50(4): 463-472.

El-Sagan, M. A. and G. A. El-Dsouky (2015). Effect of irrigation system and chitosan application on productivity of garlic under siwa condtions. Zagazig J. Agric. Res., vol 42 No. (6), 32 ref.

El-Zohiri, S.S.M. (2015). Performance Comparison of Three Alternatives for $\mathrm{GA}_{3}$ on Growth, Earliness and Total Yield of Globe Artichoke. Middle East J. Appl. Sci., 5(3): 636-644, 43 ref.

Kandil, H. (2014). Response of pea plants (Pisum sativum L.) to phosphorus levels and humic acid levels. Intern. Conf. of Agric. Eng., 6-10 July 2014, P 0136. (C.F. Computer Search CAB Abstracts).

Mohsen, A.A.M., Sabreen. K.H. A. Ibraheim and M. K. Abdel-Fattah (2017). Effect of potassium Humate, Nitrogen Biofertilizer and Molybdenum on Growth and Productivity of Garlic (Allium sativum L.). Curr. Sci. Int., 6(1): 75-85, 34 ref.

Sajid, M., A. Rab, S. Tanveer Shah, I. Jan, I. Haq, B. Haleema, M. Zamin, R. Alam and H. Zada
(2012). Humic acids affect the bulb production of onion cultivars. Afr. J. Microbiol. Res. 6 (28), 5769-5776, 21 ref.

SAS (1996). Statistical analysis system. SAS user`s guide: statistic SAS institute Inc. editors, cary, Nc.

Scott, P. (2008) Physiology and Behavior of Plants. John Wiley \& Sons Ltd, Chichester, UK.

Shams, A. S., F. A. Abo - Sedera, A. Abo El Yazied, M. M. El-Nagar and M. S. EL-Badawy (2014). Effect of foliar spray with some safety compounds on growth, productivity and quality of some strawberry cultivars. J. Plant Production, Mansoura Univ., Vol. 5 (8): 1419 - 1432.

Shehata, S.A., H. A. Hassan, A. A.Tawfik and Mervat F. Farag (2016). Effect of some stimulants and spraying amino acids on growth and chemical compostion of cucumber plants grown under greenhouse condtions. Zagazig J. Agric. Res., vol 43 No. (4), 31 ref.

Shekari, G. and J. Javanmardi (2017). Effects of Foliar Application Pure Amino Acid and Amino Acid Containing Fertilizer on Broccoli (Brassica oleracea var. italica) Transplants. Adv. Crop. Sci. Tech. 9(2): 29-33, 17 ref.

Snedecor, G.W. and W.G. Cochran (1991). Statistical Methods, $6^{\text {th }}$ ed. Iowa State University, Ames, 593 pp.

Winton, A. L. and K. B. Winton (1958). The Analysis of Food. Johan Wiley and Sons, Inc. London, P.857.

Zheng, W. and S. Y. Wang (2001). Antioxidant activity and phenolic compounds in selected herbs. Journal of Agricultural and Food Chemistry, 49: 5165-5170.

$$
\begin{aligned}
& \text { تأثير بعض المركبات الامنة و طريقة الاضافة على النمو و الانتاجية فى الخرشوف }
\end{aligned}
$$

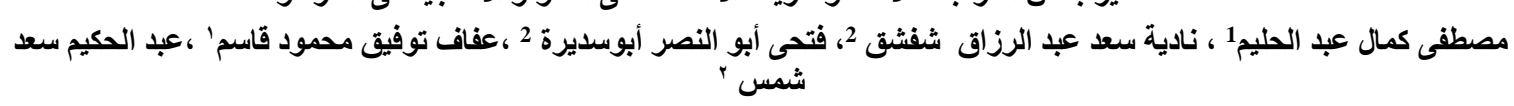

$$
\begin{aligned}
& \text { 1- قسم البطاطس و الخضر خضرية التكاثر - معهد بحوث البساتين- مركز البحوث الزر اعية - جيزة. }
\end{aligned}
$$

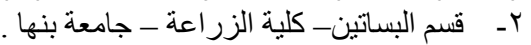

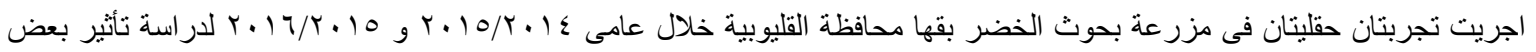

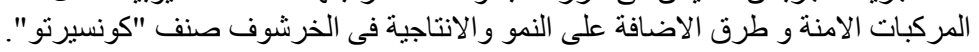

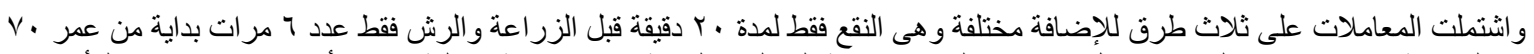

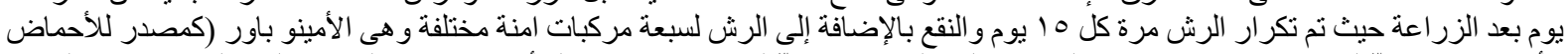

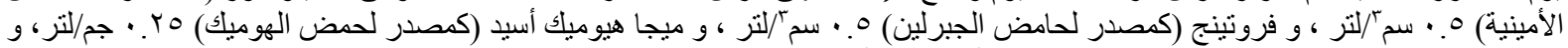

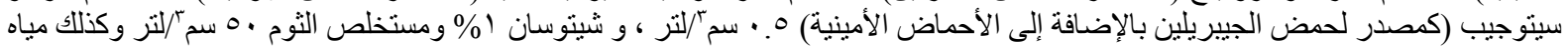

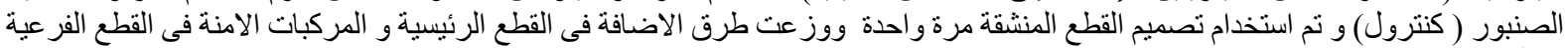

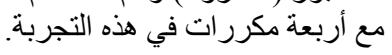

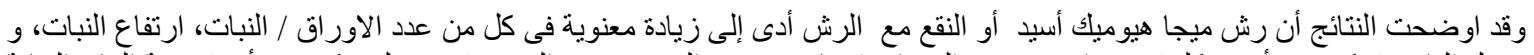

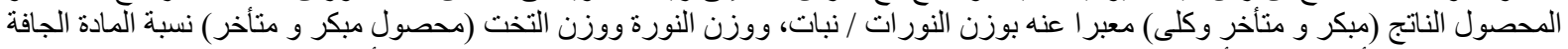

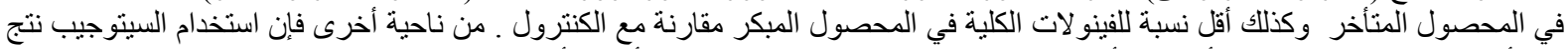

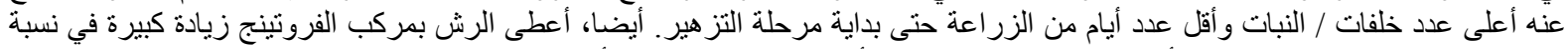

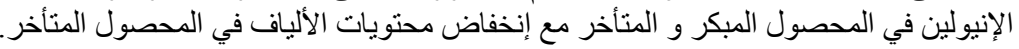

$$
\begin{aligned}
& \text { الكلمات الدالة : الخرشوف ، حض الهيوميك ، مستخلص الثوم ، شيتوسان ، جبرالين ، نقع ، رش }
\end{aligned}
$$

